\title{
Co-Producing Digitally-Enabled Courses that Promote Desistance in Prison and Probation Settings
}

\begin{tabular}{|r|l|}
\hline Journal: & Journal of Criminological Research, Policy and Practice \\
\hline Manuscript ID & JCRPP-07-2018-0023.R2 \\
\hline Manuscript Type: & Case Study \\
\hline Keywords: & $\begin{array}{l}\text { Treatment, Through-care, Offenders, Evidence based practice, } \\
\text { Management, Risk }\end{array}$ \\
\hline \multicolumn{2}{|l}{} \\
\hline
\end{tabular}


Submission to Journal of Criminological Research, Policy and Practice

\title{
Co-Producing Digitally-Enabled Courses that Promote Desistance in Prison and Probation Settings
}

\begin{abstract}
Purpose

This paper sets out an approach to innovation in criminal justice settings that gives service users a 'voice' through the co-production of digital content designed for services that promote desistance. The authors describe the benefits and challenges of involving service users in co-creating mediated digital content within a co-production framework.
\end{abstract}

\section{Approach}

This paper presents a new methodology for developing desistance-oriented programmes. We draw on a distinctive co-production exemplar within a prison setting that captures the perspectives of people who have shared their voices and we begin to explore the impact that co-production has had for them and for the service.

\section{Findings}

The testimonies of service users involved in this exemplar provide insights into the benefits and challenges of co-production in the criminal justice system more broadly.

Practical Implications

- Co-production is a credible service design strategy for developing digital services in prisons and probation

- Complementary Digital Media (CDM) provides a promising pedagogical approach to promoting desistance

- CDM enables service users to share their voice and stories to assist their peers.

- Digitally enabled courses to promote desistance can be well suited to peer support delivery models 
Originality/ Value

Complementary Digital Media (CDM) is a novel approach that uses co-production to create highly tailored content to promote desistance in discrete target groups. CDM can be used to digitalise processes within traditional Offending Behaviour Programmes (OBPs). It can also enable the development of innovative toolkit approaches for flexible use within day-to-day therapeutic conversations between service users and criminal justice staff or peer supporters. CDM thereby offers practitioners in criminal justice settings an entirely new set of evidence-informed resources to engage service users.

NB - This paper provides an exposition of the authors' views on co-producing technology that supports desistance. It is not intended to set out HMPPS policy on digital rehabilitative services nor co-production methods ${ }^{\mathrm{i}}$.

\section{Digitalisation of Justice}

Van De Steene and Knight (2017) identify a range of opportunities and challenges associated with the digitalisation of prisons. Our justice system is at the cusp of a digital revolution and expectations that all service users will engage with digital services are gaining momentum. Advancements in the use of technology in the criminal justice system (CJS) have led to suggestions that "the inevitability of digital transformation is set to shape the way justice is done and experienced" (Van De Steene \& Knight, 2017 pp. 256).

The deprivation of digital technology whilst in prison has the potential to create hidden harms by rendering people in prisons as digital 'cavemen' [or women] (Jewkes \& Johnson 2009) and leading to 'digital jet-lag' upon reentry to the community (Knight 2016). In the context of prison settings, digital services have the potential to transform people's imprisonment and their journey towards desistance ("the longterm abstinence from criminal behaviour"; McNeil, Farrall, Lightowler \& Maruna; 2012; pp.3). Knight (2015) highlights that the benefits of maintaining digital 
engagement as therapeutic and contributing to the safer custody and decency agendas. Moreover, in an academic context, Farley and Pike (2016) repeatedly evidence that digital engagement can help the learning journeys of people in prison.

Reisdorf and Rikard (2018) helpfully recommend a 'model of digital rehabilitation and reentry' to account for how people in prison transition back into the community. They recommend an adaptation of Helsper's (2012) 'basic corresponding fields model', which outlines the economic, cultural, social and personal fields. Reisdorf and Rikard (2018) argue that these need to be acknowledged when thinking about the benefits of digital interaction and use. They argue that enhancing these domains is likely to result in improved outcomes for users upon resettlement. The value of digital literacy more broadly has salience within the justice landscape. If we adopt the technological determinist view (Selwyn, 2002), enhancing people's digital skills can have important value for the citizenship and quality of life of people in prisons and probation.

The digitalisation agenda in the criminal justice system is highlighting an interesting and emerging intersection in scholarly thinking within criminology. Stratton, Powell and Cameron's definition of 'Digital Criminology' (2016) addresses the criminological study of computer and cyber crime. Whilst this definition is gaining momentum, the authors of the current article (and others ${ }^{\mathrm{ii}}$ ) are making contributions that broaden the focus to technologies that promote desistance rather than the opposite (i.e., offending). Digitalisation offers the potential to use technology to strengthen people's commitment to desistance via orchestrated evidence-informed services that reduce the damaging effects of physical conditions and transitions (including "through-the-gate") in the criminal justice system. The current case study contributes significantly to this young and emerging field.

\section{Innovating in the Justice System}

The development of digital innovations within the justice sector is met with both excitement and trepidation. As Nellis (2006) has previously argued (in a review of electronic monitoring), more can be done to ensure that technological advancements 
in the justice field make a "conscious educative effort" to support therapeutic outcomes, rather than merely reinforcing existing punitive values. Furthermore, Cheliotis (2014) warns that innovation (which could be arts-based programmes, therapeutic work or community engagement) can be perceived as 'decorative justice'. He warns that innovation can have the 'function of masking the injustices and painful nature of imprisonment behind claims of fairness, benevolence and care' (ibid.p16). These considerations are valuable when considering how digital innovation could indeed serve to reinforce punitiveness and mass incarceration - because 'innovation is not morally or politically neutral' (Graham 2017).

There is a challenge therefore (and an opportunity) to ensure that stakeholders in the justice system innovate in ways that maximise the potential of the desistance agenda. Evidence suggests that therapeutic approaches in the justice system are most likely to promote desistance if they enable participants to: form meaningful therapeutic relationships with practitioners (see Ross, Polaschek \& Ward, 2008); identify and build on strengths (McNeil, 2012); and, develop skills that allow them to lead better lives (Looman \& Abracen, 2013). We believe it is possible to address the challenge of promoting desistance by adopting an optimistic and collaborative approach to innovation, captured by Graham (2017):

Innovation is a multi-faceted topic that has the capacity to be researched and celebrated in inclusive and emancipatory ways, making the knowledge base more epistemologically open and co-produced by hearing voices, experiences and expertise that may not have been included or valued as much in the past. (Graham, 2017, pp. 206)

In line with Graham (2017), the potential risks associated with the digitalisation of prisons can be mitigated through the development of technologies in prisons and probation that explicitly promote desistance and use co-production to place service users as close to the centre of their design and implementation as possible. Bovaird (2007) defined co-production as 'the provision of services through regular, long-term relationships between professionalised service providers (in any sector) and service users and/or other members of the community, where all parties make substantial resource contributions' (pp. 849). In her pioneering work, Weaver (2013) describes 
ways of "co-producing desistance" in individual, group or community forms. In the current article, we describe a form of group co-production where service users are brought together to shape a new violence reduction service call Timewise. Focusing principally on content development, we also touch on other features of group coproduction relating to decision-making processes around policy development, delivery and the operational management of the service.

As we argue in our paper, co-producing innovation has the potential to give service users a voice and play a part in ensuring that digital services meet the needs of their peers and importantly do no harm. We explore the challenges facing the developers of technologies to promote desistance and offer some solutions via our direct use of stories and voices from service users. The evidence-base for digital co-production in the justice system remains limited. We look to contribute to the evidence-base by presenting service user reactions to being involved in the co-production of a digital service that makes a conscious educative effort to promote desistance.

\section{Co-Producing Digital Innovation in Criminal Justice Settings}

There is a rich tradition of co-production in the field of social innovation (see Voorberg, Bekkers \& Tummers, 2014). Successful examples exist of former and current service users co-developing complementary adjuncts to extant criminal justice service provision (e.g. Weaver 2013). Kristensson, Matthing and Johansson (2007) argue that engaging service users in co-production ensures that developers can make digital products and services more useable and relevant to lived experiences.

Technologies are being developed ${ }^{\mathrm{iii}}$ for use in prisons in England and Wales that employ user-centred design and co-production techniques to support service users. We outline some current exemplars of such services below:

- HMPPS has a "Digital Studio" that works to Government Digital Service (GDS) standards to create user-centred software enabling access to a range of resources via in-room (i.e., in-cell) computer terminals in two HMPPS prisons. 
- A company providing the software for the Virtual Campus (available in 95\% of prison education departments) is continually capturing user feedback to inform versions of its service user-facing e-learning platform.

- A company developing Interactive TV services for private prisons in England has been engaging service users to inform the design and content of their digital platform.

- McDougall, Pearson, Torgerson and Garcia-Reyes (2017) reported on the selfresponsibility that the "Unilink" system provides users in prison. This system enables users to order items for personal use, select meals, book visits and contact staff. The software design process routinely captures feedback from users and peer mentors who have a role in supporting their peers in getting the most out of the system. Furthermore, a workshop in one prison is employing its men to build the wing kiosks that enable users to access the Unilink system.

- A number of private companies are now offering secure software and tablet solutions developed in accordance with user-centred and iterative service design principles. These systems can deliver digital content and services in both prison and community settings.

Furthermore - outside of England and Wales - the Probation Board of Northern Ireland has an application named 'Changing Lives' which is designed for people on probation. It provides a journal, information about being on probation and signposting to mental health and addiction services (McGreevy, 2017). This innovation has been developed in conjunction with a working group, which involved service users.

To further advance the involvement of users in the development of digital services in prisons and probation, we argue that the explicit use of their stories and voices has the potential to engage, entertain and promote desistance amongst their peers. Indeed, two providers of digital "edutainment" services in prisons have enabled service users to creatively and directly share their voices with prison audiences. Firstly, the Prison Radio Association (PRA) has recording facilities in one male and one female prison, and generates content to broadcast on National Prison Radio (a radio station they set up and run). PRA has also created a mobile phone application ( 'Straightline') for people released from prison. This app has the explicit purpose of presenting inspiring service user stories to promote desistance. Secondly, WayOutTV - currently available 
in 25 prisons - provides a platform for service users to share their creative work completed during education sessions anonymously with their peers in prisons.

In addition to the services mentioned above, digitalisation within the criminal justice system also paves the way for mobile technologies that support practitioners and promote desistance amongst service users. "eHealth" services consist of technologies (e.g., web-based interventions, apps, wearables, or virtual reality) designed to improve and support health, well-being and quality of care (Van Gemert-Pijnen, Kip, Kelders, Sanderman \& Kelders, 2018). Internationally, prisons and probation services have been slower than other sectors (such as health) to adopt these kinds of services (Ross, 2018).

\section{Promoting Desistance with Technology}

In a systematic review, Kip, Bouman, Kelders and van Gemert-Pijnen (2018) identified fifty studies that captured the characteristics of a wide range of "eHealth" technologies applied in criminal justice settings. They established six categories of application and identified prominent strengths and weakness across these studies. They listed advantages of eHealth applications in criminal justice and forensic mental health settings including: increasing access to rehabilitation opportunities (e.g., overcoming physical barriers like distance or security); better fit with patient needs/ preferences/ living environment; effectiveness; efficiency; and, fidelity in delivery. Disadvantages of e-health interventions included decreases in or lack of in-person contact, ineffectiveness of treatment, costly implementations and technological malfunction.

Seventeen of the studies in Kip et al's review fell into the category titled "Interactive, Predominantly Language-Based Interventions". These interventions aim to change offence-related cognitions or behaviour, mostly via language-based information, assignments, or exercises. According to Kip et al (2018) this category of intervention can be delivered via multiple modalities (e.g., written text, videos, or audio), and is often based on theory or existing, evidence-based therapies. One example of this class of eHealth approach is the Breaking Free online drug treatment and recovery app (Elison et al., 2016). This service is being delivered in a range of contexts, including a 
private prison in England where tablets are issued to participants on a drug recovery wing during sessions that take place in a dining area.

Ross (2018) reports on the increasing prevalence of digital health interventions in health, mental health and justice systems in the shape of web-based and mobile apps. He highlights the need for evidence to report on the value and effectiveness of these digital services and he suggests that these technologies 'may be transformative or may change things very little' (ibid. p52). Ross (2018) identifies key challenges that need to be overcome to provide effective services in this space, which include:

- Adapting pre-existing approaches to work effectively in a digital environment

- Ensuring quality and genuine benefits for practitioners and service users

- Enabling access to the service and motivating people to use it

We believe that to overcome these challenges (and to be confident that services do no harm) digital services that promote desistance should be strengths-based and place service users as close to the centre of their design as possible.

\section{Complementary Digital Media (CDM)}

Complementary Digital Media is a technology-based strategy to enhance approaches intended to promote desistance ${ }^{1}$. CDM clips create a shared focus for participants and supporters and provide a starting point for service users to explore the personal relevance of specific learning objectives. CDM clips present skills is relatable situations generated by service users. For example, in one CDM clip the "Change, Accept, Let Go" skill is described. Audio, text and visuals are combined to make the character's self-talk visible in a situation where they successfully cope with angry, ruminating thoughts whilst they wait for an adjudication hearing.

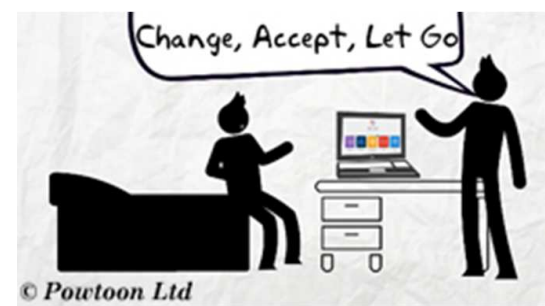

Fig 1. Screengrab from the Change, Accept, Let-Go Timewise CDM clip

\footnotetext{
${ }^{1}$ An explanation of CDM can be found here: https://youtu.be/QFWBnUYTvMk
} 
One of the authors of this article, described a digitally-enabled violence reduction toolkit (the Timewise Channel) for people serving sentences in prison consisting of 22 CDM clips accessed by men via in-room computer terminals (Morris \& Bans, 2018). The Timewise in-room Channel is currently only being piloted at one prison and the service is subject to process and impact evaluations.

CDM clips (such as those in Timewise) are designed to take advantage of a range of digital learning strategies. Firstly, CDM clips make concurrent use of audio to explain dynamic visuals in line with Mayer (2001)'s "modality principle", which dictates that audio is superior to written text when presenting complex, fast-paced information not under the learner's control. Secondly, CDM clips create connections between different elements (e.g., through colour coordination) to highlight key points. This is known as the 'signalling principle' (see Richter et al. 2016), which is particularly effective for learners with low prior knowledge. Thirdly, by presenting realistic situations which relate to the experience and situations of users - CDM clips aim to create a balance between the independent subsystems of working memory (i.e. audio, visual, and episodic; Baddeley, 2000) to aid the transfer of learning outcomes to longterm memory.

CDM clips can be hosted on a range of digital platforms for access during face-to-face therapeutic conversations and between sessions (i.e., at the participant's convenience in their own space and time).

There is some evidence that harnessing the experience and expertise of service users within therapeutic programmes has enhanced the credibility, meaning or legitimacy of those services in the eyes of participants (e.g., Morrison, Doucet \& Murray, 2006). Although examples of this within criminal justice settings are limited, Hodge, Davis, Maiden, Mann, Nidsjo, Simpson, and Reynolds (2015) provide a detailed description of co-production during the development of a computer game that enables participants to experiment with behavioural responses within risky situations (such as peer pressure) to help them make better choices. We argue that co-production initiatives like this can be important for amplifying the learning of valuable skills to promote 
desistance. In the next section, we describe a distinctive case study of CDM coproduction in the context of the Timewise Channel.

\section{Co-Producing CDM and the 4Pi Framework}

The 4Pi National Standards (NSUN, 2014) provides a framework to underpin service user involvement projects in the space of mental health service design. This framework sets out standards for good practice, measurement, monitoring and evaluating service user involvement. The 4Pi standards ensure that user involvement is characterized by 4 ' $\mathrm{P}$ 's:

- Principles - a clear focus on shared values of fairness, respect and inclusivity

- Purpose -clearly stated aims and intended outcomes from the beginning

- Presence - involving users at all levels of the service design process

- Process -how service users can get involved, being flexible and creating roles that reflects the strengths and interests of users In undertaking these the impact - or ' $\mathrm{I}$ ' is to assess if change has taken place:

- Impact - to establish how user involvement has made a difference

CDM has been co-produced with Service User Reference Groups (SURG) run in accordance with the 4Pi framework. Service users are recruited in accordance with equal opportunity selection processes. Once selected, SURG participants sign a consent form that is finalised following a preliminary SURG meeting. From the outset, participants consent to contributing to a range of activities, including:

- Creating ideas for digital content: identifying topics, skills and scenarios relevant to the aims of the service being developed. This involves developing storyboards and scripts that model the use of skills in relatable situations.

- Recording voice-overs and creating video content for CDM.

- Providing feedback on the CDM and guidance notes on how to use it therapeutically. 
- Suggesting ways to motivate intended communities to engage with the new service and designing marketing materials (posters, leaflets, promotional CDM and recognition of engagement).

- Suggesting strategies for integrating services into existing organisational structures.

SURG meetings require careful planning so that activities are coordinated in ways that enable SURG participants to make their best possible contribution. SURG members are encouraged to develop and practice skills (e.g., in the development of storyboards, the delivery of voiceovers, use of recording equipment, etc). At the end of the SURG process, participants are provided with feedback in recognition for their contributions. They are also presented with completed CDM clips prior to them being used in therapeutic services.

\section{Case Study: The Timewise Channel}

Timewise was originally piloted in 2016 as a conventional CBT-based violence reduction programme that incorporated CDM clips into face-to-face groupwork sessions. Following a review of staff and service user reactions to Timewise, the Timewise Channel was designed. A number of the original CDM clips were re-voiced by service users during a project coordinated in conjunction with the drama department at a Young Offenders Institution. The content of the Timewise Channel was uploaded by the HMPPS Digital Studio on to the "Hub" of media content accessed by service-users via in-room computers at the pilot site. The channel was launched at a single adult male prison in February $2017 .{ }^{\text {iv }}$

The content of the Timewise Channel consists principally of CDM clips designed to promote CBT-based rehabilitative skills and concepts modeled in realistic and relatable scenarios (Morris \& Bans, 2018). Timewise CDM clips provide microlearning experiences that aim to address specific learning outcomes (aligned to concepts in existing HMPPS Offending Behaviour Programmes; OBPs). The Timewise Channel makes use of CDM clips within a blended-learning approach, where CDM is used to get conversations started between participants and supporters 
(regardless of their expertise and experience). The conversations form the basis of effective working alliances and promote better coping with the stressors of prison life. The Timewise Channel can also be accessed independently as a self-directed learning activity completed in the relative privacy of the participant's room.

\section{Co-Producing CDM for the Timewise Channel}

The Timewise Channel was iterated substantially in conjunction with service users (people serving prison sentences at the pilot site) and the analysis of usage data supplied by the HMPPS Digital Studio. Usage data enabled hypotheses about the apparent success or otherwise of particular pieces of content to be tested and explored during SURG meetings. SURG members reviewed the initial Timewise CDM content themselves and provided feedback. The a psychologist in training then coordinated them in supporting their peers in accessing the Timewise Channel and applying their learning to their own lives. SURG members (some of whom were also peer supporters) were then asked for feedback on a weekly basis during SURG meetings. In line with the SURG consent form and the 4Pi standards, SURG meetings explored issues underpinning violent conflict and daily stressors experienced at the pilot site. SURG members were asked to discuss coping strategies for coping with these challenges. These discussions created the basis for new CDM clips that could be added to the Timewise Channel by the HMPPS Digital Studio. The co-production of CDM involved service users being coached in the creation of storyboards for scenarios, as well as them developing scripts and delivering voiceovers to enable animations to be produced. Animations were continually presented back to SURG members and iterated in line with their feedback and that from other stakeholders. An example of a co-produced animation includes the exploration of different styles of self-talk to help men self-manage aggressive thoughts presented in a scenario where a man's medication was confiscated.

In addition, SURG meetings explored ideas to improve the design of the Timewise service generally. In particular, SURG members made a number of suggestions as to how they could support participants (in their role as peer supporters) so that participants could get the most out of accessing CDM clips. Peers supporters edited Timewise materials and produced new ones. 


\section{The Co-Creator Perspectives}

A researcher with no prior involvement with the Timewise Channel facilitated a group discussion $^{\mathrm{v}}$ with SURG members. This discussion consisted of six peer mentors who were responsible for creating CDM content and supporting their peers in accessing the Timewise Channel. An audio recording of the discussion was transcribed. A content analysis was then undertaken to code the relevance of interactions within the discussion to the different elements of the 4Pi Framework. This analysis is summarised below to set out how the model of co-production contributed to the development of the CDM. We recognise that this only reflects a small sample in research terms and our intention was not to achieve points of generalizability. Instead we wanted description and reflection that was collected by an independent person.

\section{Principles}

All the members of the group acknowledged that their role was integral to the creation of the CDM clips. As informed users of the prison system they felt they had valuable and credible knowledge to share during this process. For example, understanding that CDM was an important complementary tool especially for those who liked to learn through visual methods. These clips enabled the SURG members to help their peers 'marry up the video with the things that affect them' whilst in prison.

It is also evident that the role of the members was not just about providing feedback it was about idea development from the onset of the project- 'how it could be pushed and how we can move it forward and what we can do'. As co-creators, their involvement helped to advocate their products to support the service. This meant that their role as champions for the service more broadly was enhanced. Impetus for the development of ideas for the clips involved all stakeholders. The role of the facilitator was crucial to coordinating the group.

\section{Purpose}

Whilst the purpose of the group had been clearly defined from the onset of the project the development of materials and content for the CDM clips was shaped collaboratively. Planning and coordination was important and space was provided for 
regular and frequent meetings. These meetings facilitated discussion and the outcome was idea-production, which then translated into the creation of the CDM clips.

\section{Presence}

Their inclusion in this process was considered valuable and they reflected on the criteria for membership of the group. They listed skills and qualities that enabled the group to function and help deliver the CDM clips. For example 'being able to listen', 'respecting each other, letting others have their say'. They identified as a bridge or mediator between men and peer supporters or prison staff (including the psychology team). This was important because 'some men are uncomfortable talking to staff directly so we are the in-between'. This responsibility, they felt, was not to be taken lightly and carrying voices on behalf of the prison community was a matter of trust. Demonstrating trust and reliability was important because they suggested that 'you have got to make sure you have got that bit of integrity about you'.

\section{Process}

The production of the CDM materials to support Timewise relied heavily on group discussions with SURG to galvanise collaboration. Their input and ideas had to be realised within the context of the prison setting. So for example much of their input was centred around preparing storyboards for each of the clips. The facilitator of the group had to put together the film away from the prison premises but they followed the agreed narrative based on these planning meetings. Some of the men were frustrated that animation had to be used instead of video footage as they felt 'it will be realistic with real men in it'. They recognised the constraints of this and argued that:

'if you try to perfect it from day one you might have still been trying to make the video right now...So getting it out there and you can really say the stick men are just like the skeleton version of the video'.

As a solution, they believed that recording the men's voices would enhance the credibility and authenticity of the materials - 'you can hear it's real people'- people who were experiencing prison. They recognised that the animated route enabled them to produce materials quickly and cost effectively. This method enabled the creation of 9 of the 22 Timewise CDM clips over a relatively short time and the group members 
believed that this was only possible 'through feedback' mechanisms. The testing and script writing was led by the SURG members and the facilitator worked to ensure that content was appropriate in terms of communicating the right messages in line with the underpinning framework of the service. In addition, materials were vetted to ensure content was secure and was inclusive to all potential users.

\section{Impact}

The group members talked vividly about gaining trust and responsibility. They viewed their contribution as 'giving something back' and being able to 'achieve something'. They felt that contributing to the desistance journey by co-producing these materials and supporting their peers 'feels good to try and help people especially I have got the same experiences as that person.' One individual described how his involvement meant he could:

'connect with people because I think the way they think and try and help them come away from that. So for me that's the reason why I am doing this job, not for no red band or anything like that it's just to help people...'

For some members their involvement in the SURG has triggered aspirations to continue supportive and transformative roles when they leave prison. Furthermore their experience in developing CDM materials has sparked new ideas and aspirations for further digital development and desistance support. Their ideas included video blogging or vlogging where users could share their stories of success with the prison community. One individual highlighted that the potential for co-created CDM has wider potential- ' it could be another thing...' and not just specific to the Timewise service. They perceived technology as an opportunity to push positive messages to people in prison and the availability of in-room technology could create a digital community where experiences could be shared. This thirst for innovation and creativity is accelerated by their direct involvement with the SURG. It was also evident that their roles within the SURG enabled personal growth and membership to the rehabilitative culture. One individual explained how his interests were growing and wanted to be involved in the creation of the prison's newsletter. 


\section{Reflections and Future Directions}

The authors of this article would like to make some practical suggestions for replicating this model of working. There were challenges and much of this pertained to working within a prison setting. For example coordinating meetings to generate ideas and produce content can be challenging. Working within a tight prison regime means that facilitators are restricted to strict timings based on unlock and lock up of men. This means facilitators need to be organized and having clear objectives are advised for such meetings. Furthermore support and buy-in from senior managers is also valuable. Securing agreements with SURG members was then possible to allow for time to be used away from the group meetings also. Recruiting the right individuals is difficult and ensuring inclusivity is important. Co-production can take much longer and thus can be more resource intensive. However following the $4 \mathrm{Pi}$ framework can ensure that success is achieved. Centering the voice of others can take many guises such as gaining feedback and contributing directly to the creation of materials and services. It is evident from this exemplar that practical involvement in both creation and delivery helps participants to assist in transformation not only for the prison community but also themselves.

Considerably more empirical work needs to be done to investigate the implementation and the efficacy of the Timewise Channel. Notwithstanding, the CDM clips developed to support Timewise have served as a valuable prototype to enable us to explore the benefits of using technology to complement existing therapeutic approaches and to create new ones. This has wider implications internationally for services within corrections and beyond. We are looking to use CDM in two ways:

1. To complement conventional Offending Behaviour Programmes by integrating digital adjuncts into existing face-to-face sessions and promoting consolidation between sessions. The basic skills modelling undertaken by CDM clips provide examples of what success might look like for participants and thereby helps facilitators (working within the risk-need-responsivity model) to provide strengths-based learning experiences to participants.

2. To form the basis of toolkits that enable access to CDM to support rehabilitative conversations with a range of individuals who are out of scope for an accredited programme offer. Toolkits enable supporters and frontline 
Digitalisation offers the criminal justice system the opportunity to orchestrate a range of evidence-informed services - designed to improve well-being and the quality of human interactions - so that the psychological and reintegration needs of people who have committed crime can be met. The co-production methods showcased in the Timewise project provide an example of how voice and action can be brought together to pursue these aims. Creating digital services with service users increases the chances that user needs can be understood and ultimately met. We believe that explicitly enshrining the stories and voices of users within these services holds the key to making them accessible. Furthermore, centring services like Timewise around user voices means that content and rehabilitative visions can be co-owned with co-creators who have had a genuine opportunity to 'give something back' - an experience which may in turn form a key component of their pathway to desistance.

\section{References}

Baddeley, A.D. (2000). The episodic buffer: a new component of working memory? Trends in Cognitive Science, 4 (11), 417-23.

Bovaird, T. (2007). Beyond engagement and participation: User and community coproduction of public services. Public Administration Review, 67(5), 846-860.

Cheliotis, L. (2014). Decorative justice: Deconstructing the relationship between the arts and imprisonment. International Journal for Crime, Justice and Social Democracy, 3(1), 16-34. 
Farley, H., \& Pike, A. (2016). Engaging prisoners in education: reducing risk and recidivism. Advancing Corrections: Journal of the International Corrections and Prisons Association, 1, 65-73.

Graham, H. (2017). Innovation and criminal justice: Editorial introduction to the Special Issue on 'Innovation'. European Journal of Probation, 9(3) 203-209.

Hodge, P., Davis, J., Maiden, N., Mann, B., Nidsjo, A., Simpson, A., et al. (2015). StreetWise: a valid ecology for a serious game in a secure forensic mental health setting. Procedia Computer Science, 63, 252-259.

Jewkes, Y., \& Johnston, H. (2009). 'Cavemen in an Era of Speed-of-Light Technology': Historical and Contemporary Perspectives on Communication within Prisons. The Howard Journal of Crime and Justice, 48(2), 132-143.

Knight, V. (2005). Remote Control: The role of TV in prison. London Palgrave Macmillan

Knight, V. (2015). Some observations on the digital landscape of prisons today. https://www.dora.dmu.ac.uk/bitstream/handle/2086/10866/Digital\%20Landscape \%20 of $\% 20$ Prisons $\% 20 \mathrm{PSJ} \% 20 \mathrm{Knight}$.pdf? sequence=1\&isAllowed=y. Accessed 06/09/18.

Knight, V., \& Tighe, S. (2017). Should the public be listening to prison radio programmes? An exploration of prison radio in Sweden and North America. Prison Service Journal, 234, 31-38.

Kristensson, P., Matthing, J., \& Johansson, N. (2008). Key strategies for the successful involvement of customers in the co-creation of new technology-based services. International journal of service industry management, 19(4), 474-491. 
Looman, J. and Abracen, J. (2013). The risk need responsivity model of offender rehabilitation: is there really a need for a paradigm shift? International Journal of Behavior and Consultation Therapy,. 8(3), 30-6.

Mayer, R.E. (2001), Multimedia Learning, Cambridge University Press, New York, NY.

McDougall, C., Pearson, D., Torgerson, D., \& Garcia-Reyes, M. (2017). The effect of digital technology on prisoner behavior and reoffending: a natural stepped wedge design. Journal of Experimental Criminology. DOI: 10.1007/s11292-017-9303-5

McGreevy, G. (2017). 'Changing Lives': Using technology to promote desistance. Probation Journal, 6(3),187-190.

McNeill, F. (2012). Four forms of 'offender' rehabilitation: Towards an interdisciplinary perspective. Legal and Criminological Psychology, 17(1), 18-36.

McNeill, F., Farrall, S., Lightowler, C., and Maruna, S. (2012) How and why people stop offending: discovering desistance. Institute for Research and Innovation in Social Services. http://eprints.gla.ac.uk/79860 accessed 06/09/18.

Morris, J., \& Bans, M. K. (2018). Developing digitally enabled interventions for prison and probation settings: a review. Journal of Forensic Practice, 20 (2), 134-140.

Nellis, M. (2006). Surveillance, Rehabilitation, and Electronic Monitoring: Getting the Issues Clear. Criminology \& Pubic Policy, 5(1), 103-108.

Reisdorf, B. C., \& Jewkes, Y. (2016). Locked sites: cases of Internet use in three British prisons. Information, Communication \& Society, 19(6), 771-786.

Richter, J., Scheiter, K., \& Eitel, A. (2016).“Signalling text-picture relations in multimedia learning: a comprehensive meta-analysis”, Educational Research Reviews, 17, 19-36. 
Ross, E. C., Polaschek, D.L.L., \& Ward, T. (2008). The therapeutic alliance: A theoretical revision for offender rehabilitation. Aggression and Violent Behavior, 13, $462-480$.

Ross, S. (2018). Policy, practice and regulatory issues in mobile technology treatment for forensic clients. European Journal of Probation, 10(1), 44-58.

Selwyn, N. (2012). Making sense of young people, education and digital technology: the role of sociological theory. Oxford Review of Education, 38(1), 81-96.

Stratton, G., Powell, A., \& Cameron, R. (2017). Crime and Justice in Digital Society: Towards a 'Digital Criminology'?. International Journal for Crime, Justice and Social Democracy, 6(2), 17-33.

Van De Steene, S., \& Knight, V. (2017). Digital transformation for prisons:

Developing a needs-based strategy. Probation Journal, 64(3), 256-268.

Van Gemert-Pijnen, L., Hanneke, K., Kelders, S.M., \& Sanderman, R. (2018).

Introducing eHealth. In: L. van Gemert-Pijnen, S. M. Kelders, K. Hanneke, \& R. Sanderman (Eds). eHealth Research, Theory and Development: A Multi-Disciplinary Approach. Routledge, Abingdon.

Weaver, Beth (2013) Co-producing desistance: who works to support desistance. In: Understanding penal practice. Routledge Frontiers of Criminal Justice . Routledge, Abingdon.

${ }^{\mathrm{i}}$ We acknowledge that we have to be sensitive and responsive to the service's perspective and have to balance delicately and diplomatically how this article is written. As a result the innovation described here has been peer reviewed by not only scholars but practitioners and managers also.

ii Emerging digital scholars also include Steven Van De Steene, Bianca Reisdorf, Hannah Graham, Stuart Ross, James Tangen. This list is not exhaustive. iii There are examples of coproduction internationally. One example is Tighe and Knight (2017), which highlights case studies of prison radio and the role of participant voices in radio production.

${ }^{\text {iv }}$ The Timewise Channel workbook has since been completed by 150 participants at the pilot site and the initiative is currently subject to a process evaluation.

${ }^{\mathrm{v}}$ Independent research was conducted by De Montfort University, UK. Interviews and group discussions were undertaken with all stakeholders. 